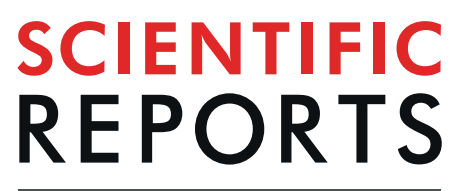

\title{
Real-time Optical Dimensional Metrology via Diffractometry for Nanofabrication
}

\author{
Guy L. Whitworth ${ }^{1,3^{*}}$, Achille Francone ${ }^{1}$, Clivia M. Sotomayor-Torres ${ }^{1,2}$ \& Nikolaos Kehagias ${ }^{1}$ \\ Surface patterning technologies represent a worldwide growing industry, creating smart surfaces and \\ micro/nanoscale device. The advent of large-area, high-speed imprinting technologies has created \\ an ever-growing need for rapid and non-destructive dimensional metrology techniques to keep pace \\ with the speed of production. Here we present a new real-time optical scatterometry technique, \\ applicable at the mesoscale when optical inspection produces multiple orders of diffraction. We \\ validate this method by inspecting multiple silicon gratings with a variety of structural parameters. \\ These measurements are cross-referenced with FIB, SEM and scanning stylus profilometry. Finally, \\ we measure thermally imprinted structures as a function of imprinting temperature in order to \\ demonstrate the method suitable for in-line quality control in nanoimprint lithography.
}

Smart surfaces with nano- and micro- scale features find application in a wide variety of sectors in modern society: electronics ${ }^{1}$, security ${ }^{2}$, photonics ${ }^{3}$, micro-optics, micro- fluidics ${ }^{4}$ and biomedicine ${ }^{5}$. High-throughput NIL technologies such as roll-to-roll, roll-to-plate, and step-and-repeat enable high-volume production of such devices and surfaces ${ }^{6-9}$. For quality control purposes, inspection technologies are required to be compatible with these high-speed patterning technologies, i.e., fast and suitable for large area critical dimension (CD) measurements ${ }^{10-13}$. Traditional powerful CD technologies such as scanning electron microscopy (SEM) and atomic force microscopy (AFM) have serious drawbacks for such an application. They are hard to insert into a production, comparatively slow, require precise alignment and are potentially destructive. For this reason, a variety of non-imaging optical technologies are used for inspection having the advantage of being fast and non-destructive.

Simple spectroscopic techniques such as reflectivity, absorption, fluorescence or Raman scattering ${ }^{14,15}$ can be used to determine the amount of a material present which in turn can be related to film thickness. However, for more complex structural determination, optical scatterometry has become a widely investigated and adopted technique ${ }^{16-18}$. Scatterometry techniques use scattered light from a surface as a function of a variable such as angle-of-incidence or wavelength. This surface scattering response is then compared to a library of simulated data created by electromagnetic (EM) modelling techniques to fit the measured response to a computational prediction a method known as inverse problem solving. Ellipsometry is an example of a powerful scatterometry technique utilising elliptical phases which can determine thin-film optical constants and layer thicknesses down to several nanometres ${ }^{19}$. For nanoscale CD metrology, angle- and or wavelength- dependant reflectometry is utilised, whereby the dependence of the reflected intensity of an incident beam ( $0^{\text {th }}$ order diffraction $)$ can be fitted to an EM library, typically computed by rigorous coupled-wave analysis (RCWA $)^{17,20-26}$. Coherent-Fourier scatterometry is a more advanced version of this technique whereby a Fourier image can be taken of the target structure using a CCD in order to obtain the reflected angle dependence in a signal shot ${ }^{16,18,27}$.

For micro-scale NIL applications where multiple orders of diffraction exist, as is the case for diffractive optical elements or smart medical surfaces ${ }^{5}$, we present a scatterometry method, advanced upon our previous work ${ }^{28,29}$, which analyses the diffraction efficiency of all collected diffractive orders simultaneously and compares this data to a finite-difference frequency-domain (FDFD) EM library. Diffraction data in our experiment was collected with a CCD (Fig. 1(a)) and normalised to the 0th order reflection using a separate photodiode to monitor the $0^{\text {th }}$ order power. This data is then compared to the EM library using a $\chi^{2}$ minimisation process to compare the experimental data to the computed model to yield the top-width, $w$, height, $h$, and SWA, $\theta$, measured in real-time (Fig. 1(b)). Figure 1(c) depicts a typical unit-cell used in the FDFD simulations for generating the metrology

${ }^{1}$ Catalan Institute of Nanoscience and Nanotechnology (ICN2), CSIC and BIST, Campus UAB, 08193, Bellaterra, Barcelona, Spain. ${ }^{2}$ Institucio Catalana de Recerca i Estudis Avancats (ICREA), 08010, Barcelona, Spain. ${ }^{3}$ Present address: Institut de Ciències Fotòniques (ICFO), Mediterranean Technology Park, Avinguda Carl Friedrich Gauss, 3, 08860, Castelldefels, Barcelona, Spain. *email: guy.whitworth@icfo.eu 


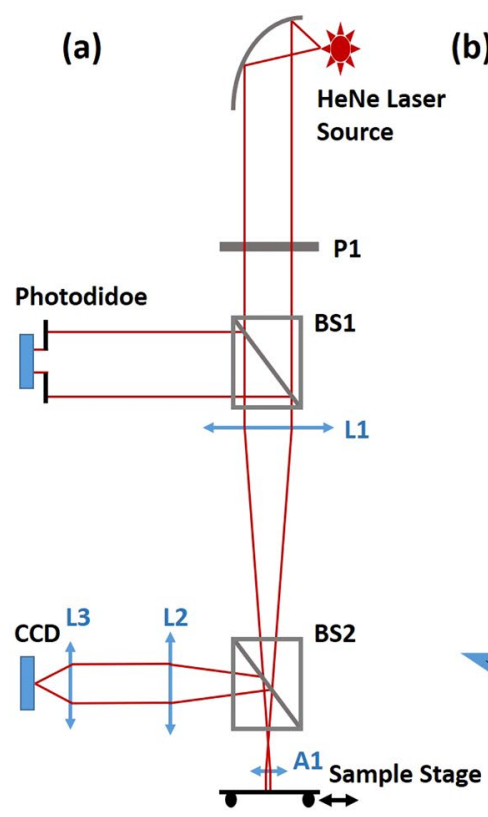

(b)

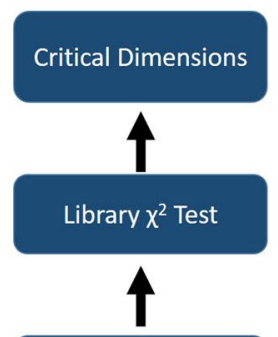

Diffraction Data

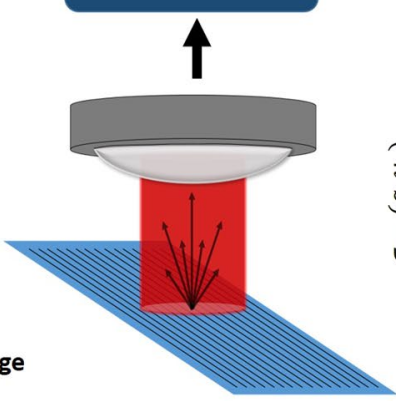

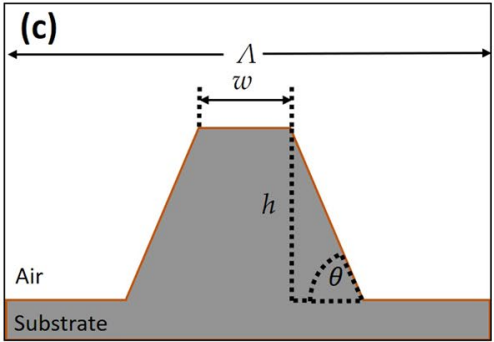

(d)

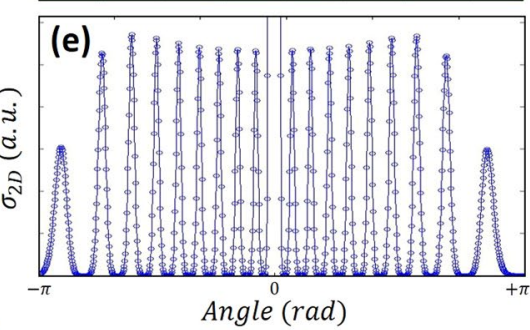

Figure 1. Experimental setup, metrology workflow and simulations. (a) An optical diagram of the diffractometry setup indicating the asphere(A1), polariser(P1) and the various lenses(L) and beamsplitters (BS). The CCD is used to collect the diffraction pattern and the photodiode for detecting the power of the $0^{\text {th }}$ order reflected mode. (b) Workflow diagram explaining the metrological process of diffractometry. (c) Schematic of simulated unit cell indicating the period, $\Lambda$, top-width, $w$, height, $h$, and side-wall angle $\theta$. (d) Example nearfield response $\left(\mathrm{E}_{\mathrm{z}}\right)$ of an incident Gaussian field on seven periods of a microstructure. The reflected response is then used to compute the far-field diffraction pattern (e) using Eq. 1.

libraries. The aforementioned structural parameters for measurement are indicated along with the grating period, $\Lambda$, which remains constant in the simulations.

\section{Results}

Diffraction pattern simulations. Careful choice of computational parameters is required when computing the diffraction libraries as an inverse-problem measurement is only as good as the quality of the simulations. In order to predict a full spatial diffraction pattern, one must first compute the near-field response and then project this into the far-field using an equivalence principle relation (Eq. 1) resulting in a diffraction pattern ${ }^{30}$.

$$
\sigma_{2 D}(\phi) \propto\left|\oint_{L}\left\{\eta_{0}\left(n_{x}^{\prime} H_{y}-n_{y}^{\prime} H_{x}\right)-\left(n_{y}^{\prime} \sin \phi+n_{x}^{\prime} \cos \phi\right) E_{z}\right\} e^{i k r^{\prime} \cos \left(\phi-\phi^{\prime}\right)} k d l^{\prime}\right|^{2}
$$

This is a rectangular closed-loop line integral along the $x$ and $y$ edges of the simulation where the near-field response of the electric $(\mathrm{E})$ and magnetic $(H)$ fields are related to the $2 \mathrm{D}$ scattering cross-section, $\sigma_{2 D}$ in the far-field as a function of angle, $\phi$.

Initial attempts to simulate diffraction patterns involved a single unit cell with periodic boundary-conditions. The resulting near-field was then stitched together with itself to be used for far-field projection. This method produced unrealistic diffraction patterns and was arbitrarily dependant on the number of repetitions chosen of the unit cell. The prevailing, more successful method was to simulate several unit cells $(>7)$ and use an incident Gaussian field, surrounded by perfectly-matched layers. Whilst the simulated beam sizes $(\sim 20 \mu \mathrm{m})$ were not accurate to the beam waist of the experiment $(\sim 200 \mu \mathrm{m})$ it was observed that the simulated diffraction pattern converged after a sufficient number of unit cells (5-7) was illuminated. An example of such a near-field FDFD simulation and resultant diffraction pattern is shown in Fig. 1(d,e).

In total, five sets of samples were fabricated to test the diffractometry tool, summarised in Table 1 below. Four were fabricated by e-beam lithography and one set via thermal nanoimprinting. Each set was designed to vary different structural parameters over different ranges (see supporting information Table S1) to test the full capabilities of the metrology technique. All structures were scanned underneath the inspection beam using an automatic translation stage with a speed of $1 \mathrm{~mm} / \mathrm{s}$.

Step-wise structure. The initial sample tested was a one dimensional, $40 \mathrm{~mm}$ long silicon grating with a $6 \mu \mathrm{m}$ period, designed to have the line-width vary over a small range $(\sim 50 \mathrm{~nm})$ in a step-wise manner over two different sections (shown in the schematic Fig. 2(a)). Figure 2(b,c) show the resultant top-width and height from the $\chi^{2}$ minimisation (blue and red respectively) obtained via diffractometry (SWA data can be found in supporting information Fig. S1). The coloured shaded area is the spread of possible results which are within one sigma of the $\chi^{2}$ minimum, calculated from the raw signal variation. That is to say that if there are defects or the structural 


\begin{tabular}{|l|l|l|l|l|l|l|}
\hline $\begin{array}{l}\text { Sample } \\
\text { Code }\end{array}$ & Design & $\begin{array}{l}\text { Period } \\
(\boldsymbol{\mu m})\end{array}$ & $\begin{array}{l}\text { Area }(\mathbf{m m} \\
\mathbf{x ~ m m})\end{array}$ & $\begin{array}{l}\text { No. of } \\
\text { Samples }\end{array}$ & Material & $\begin{array}{l}\text { Key Structural } \\
\text { Parameter }\end{array}$ \\
\hline S & Step-Wise & 6 & $40 \times 5$ & 1 & Silicon & Width \\
\hline T & Tapered & 5 & $70 \times 0.5$ & 1 & Silicon & Width \\
\hline H & Height Variation & 5 & $20 \times 20$ & 3 & Silicon & Height \\
\hline D & Defective (See SI) & 5 & $20 \times 20$ & 4 & Silicon & All \\
\hline N & Nanoimprinted & 5 & $20 \times 20$ & 9 & PMMA & Height \\
\hline
\end{tabular}

Table 1. Sample description and key parameters.

(a)

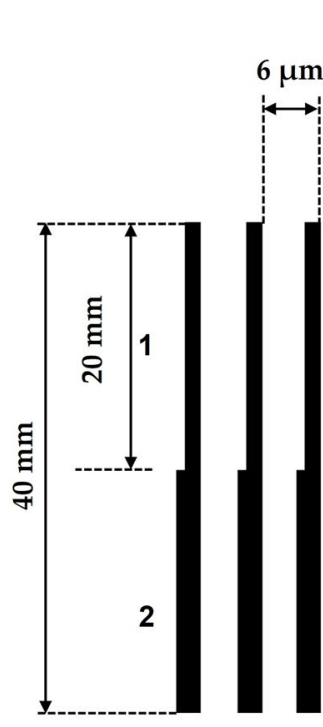

(b)

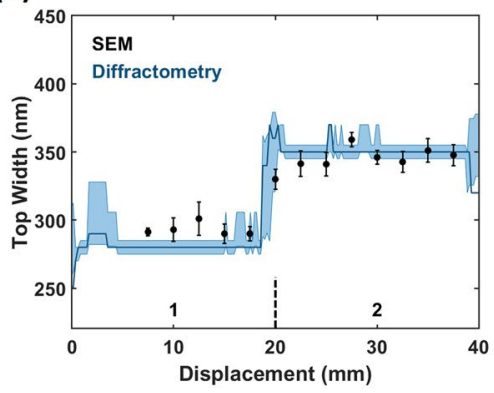

(d)

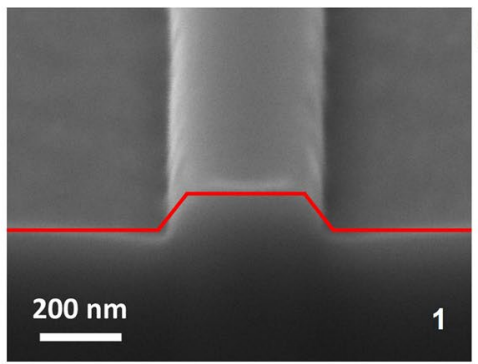

(c)

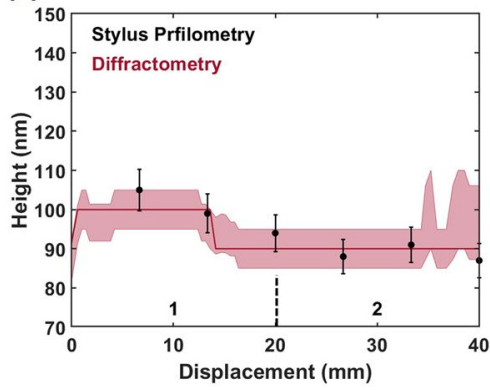

(e)

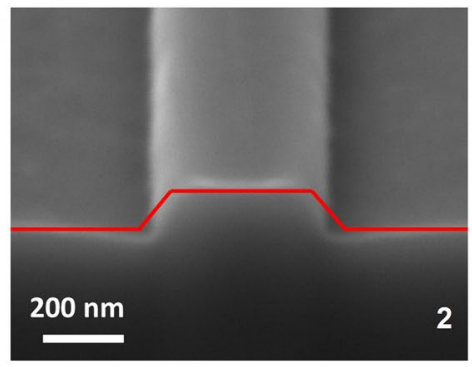

Figure 2. Step-wise structures. (a) Structural design of the $40 \mathrm{~mm}$ long step-wise structure with sections 1 and 2 indicated. (b,c) Diffractometry results for the top-width and height (blue and red). The black points represent the respective cross-referencing measurements of SEM and stylus profilometry. (d,e) SEM images of FIB cut cross-sections of the sections with an overlay accurate to the average diffractometry results respectively from each section.

dimensions are changing rapidly, the standard deviation increases and there will be multiple degenerate outcomes from the $\chi^{2}$ minimisation process within the uncertainty of the experiment.

A clear stepwise behaviour can be observed in the top-width data (blue points in Fig. 2(b)) between the two sections of the grating. Top-down SEM images were taken every $2.5 \mathrm{~mm}$ along the grating to measure the top linewidth (black points) as a cross-reference. This image analysis was performed by eye and the error bars are computed as the standard deviation between five separate measurements. The two sections can be seen to be stable in structural parameters and match closely to the diffractometry results. There is a small $20 \mathrm{~nm}$ discrepancy at $12.5 \mathrm{~mm}$ displacement as accompanied by a large standard deviation as measured widths via SEM indicating a defective region. The height data in Fig. 2(c), plotted in the same manner as that of the top-width, was cross-referenced using stylus profilometry. The data shows that the height of the structure varied between 90 and $100 \mathrm{~nm}$ along the gratings as expected from uniform etching.

To further verify the accuracy of the diffractometry, FIB cuts were made in the silicon lines. Angled SEM images (Fig. 2(d,e)) were then taken of these the separate cuts and the averaged results from the diffractometry data for each section was then overlaid onto the SEM images (red lines). The height and width data are seen to overlap well with the cross-sectional profile of the grating line, however, the SWA differs. This is assigned to the unexpected presence of over-etching at the base of the S-series gratings and a certain level of corner rounding is present in the samples. Top and bottom corner roading radii as extra simulation parameters have been omitted for simplicity in the development of the technique, as the total simulation time is increases linearly to the volume of the input n-dimensional parametric data cube.

Tapered grating structure. To further test top-width sensing, a $70 \mathrm{~mm}$ long right-angled Tapered grating was fabricated with a period of $5 \mu \mathrm{m}$ and designed to have the width vary from $200 \mathrm{~nm}$ to $4.8 \mu \mathrm{m}$ (Fig. 3(a)). The tapered structures were passed through the optical inspection beam of the diffractometer as with the previous case to obtain real-time top-width measurement, shown in Fig. 3(b) (blue data). A clear linear trend could be observed as expected from the tapers (height and angle data can be found in supporting information Fig. S2). The measurements of the top-width were noisy in the $0-15 \mathrm{~mm}$ displacement region, deviating from the linear trend, 
(a)

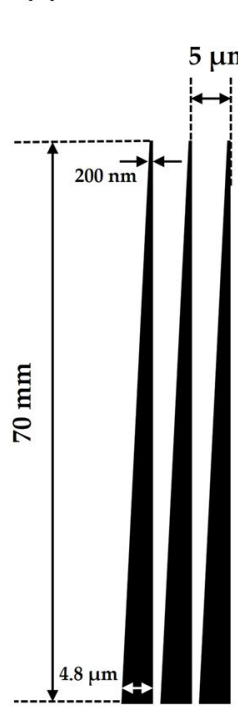

(b)

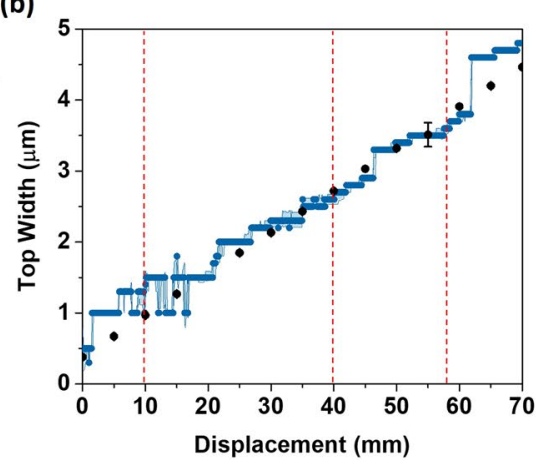

(d)

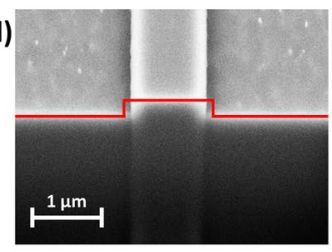

(c)

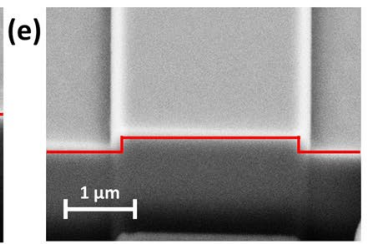

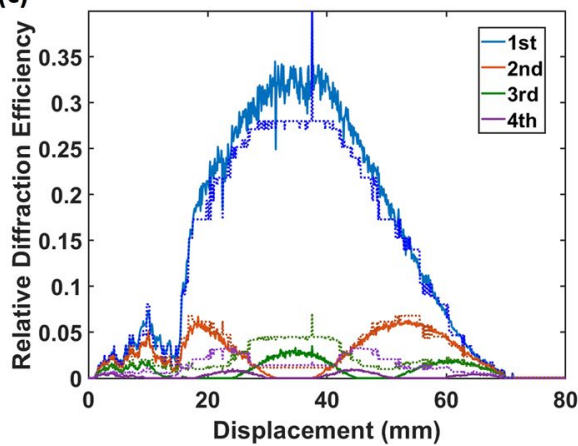

(f)

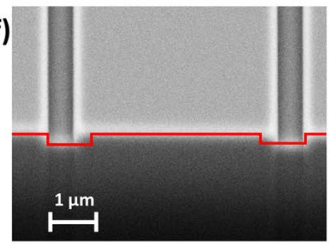

Figure 3. Tapered structures. (a) $70 \mathrm{~mm}$ long right-angled tapered design of gratings. (b) Diffractometry top-width results (blue) with SEM data (black points). Dotted red lines indicate approximately where FIB cuts were made. (c) Raw (solid) and fitted (dotted) diffraction data for the first 4 orders of diffraction collected as a function of the displacement along the tapers. (d-f) SEM images of FIB cut cross-sections the indicated locations with red overlays obtained from the diffractometry data.

also reflected in the measured relative diffraction efficiency shown Fig. 3(c) (solid lines). The noisy raw signal in this section was due to a residual polymer layer on the surface of the structure which contributed to increased scattering as seen in the optical images in supporting information Fig. S3. As before, width measurement data obtained via SEM images has been overlaid on the diffractometry data in Fig. 3(b) to verify the results (black points). Also shown in Fig. 3(c) is the resultant "fitted" diffraction efficiencies (dotted lines) as predicted by the FDFD solver.

Figure 3(d-f) show three cross-sectional FIB cuts taken along the tapered gratings at the locations indicated by the red dotted lines in Fig. 3(b), the positions of which were determined by optical microscope inspection. Note that these three positions do not necessarily coincide with the top-down SEM measurements (black points). The redline overlays are the parameters determined by diffractometry at these three locations. The height of the structure matches well with the cross-sections, consistently determined to be $250 \mathrm{~nm}$ across the majority of the structures (Fig. S2(a)). Additionally, a 90 SWA was predicted (Fig. S2(b)) in agreement with the steep side-wall angles seen by top down SEM images of the structures.

Height sensing. To test the robustness of this method against height variation, three linear gratings were fabricated with different etch depths, $\mathrm{H} 1, \mathrm{H} 2$ and $\mathrm{H} 3$. Copies of these structures were also designed with $10 \%$ of their lines missing to simulated fabricated defects, D1, D2 and D3 the results of which can be found in supporting information Fig. S4. Figure 4(a-b) show the diffractometry results and an SEM image for the sample H1. Each sample was scanned separately for inspection and the three separate results were stitched together at 2 and $4 \mathrm{~mm}$ in Fig. 4(a).

Comparing samples $\mathrm{H} 1, \mathrm{H} 2$ and $\mathrm{H} 3$, it is observed that when the inspection beam illuminates the sample, a constant height is measured with a small degree of uncertainty from the $\chi^{2}$ minimisation. Moving from samples $\mathrm{H} 1, \mathrm{H} 2$ and $\mathrm{H} 3$ the diffractometer measures heights of $440 \mathrm{~nm}, 360 \mathrm{~nm}$ and $110 \mathrm{~nm}$ respectively, using an EM library with a parametric resolution of $\pm 10 \mathrm{~nm}$. The black points and error bars show the height data as measured by stylus profilometry, $430 \mathrm{~nm}, 305 \mathrm{~nm}$ and $155 \mathrm{~nm}( \pm 10 \mathrm{~nm})$ respectively for $\mathrm{H} 1, \mathrm{H} 2$ and $\mathrm{H} 3$.

The obtained top-width (Fig. 4(b)) between all samples showed a relatively constant value, 140 or $160 \mathrm{~nm}$, which corresponds well with SEM image analysis of the top $(148 \pm 9 \mathrm{~nm})$. The combined SEM and stylus profilometry analysis for all samples estimates the SWA to be $78 \pm 3^{\circ}$. This is in good agreement with diffractometry for $\mathrm{H} 1$ measuring a SWA of $80^{\circ}$. However, $\mathrm{H} 2$ and $\mathrm{H} 3$ deviate, with diffractometry measuring side-wall angles of $70^{\circ}$ and $30^{\circ}$ respectively (Fig. $4(\mathrm{c})$ ). This inaccurate prediction of the SWA is believed to be the cause of why there is a 35 and $20 \mathrm{~nm}$ discrepancy between diffractometry and profilometry in samples $\mathrm{H} 2$ and $\mathrm{H} 3$ respectively. Most likely the simple trapezoidal modal is not describing the structures accurately enough, leading to inexact predictions of all the parameterised dimensions.

Thermal nanoimprinted PMMA structures. A set of nine poly(methyl methacrylate) (PMMA) films was patterned by thermal nanoimprint lithography (NIL) using the structure $\mathrm{H} 2$ as the master. The imprinting temperature between the samples was varied from $120^{\circ} \mathrm{C}$ to $200^{\circ} \mathrm{C}$ in $10^{\circ} \mathrm{C}$ steps, with the imprinting time kept 


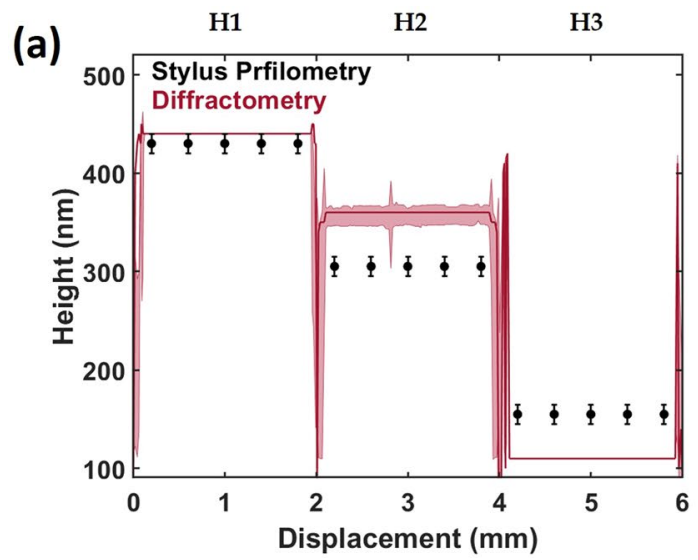

(b)

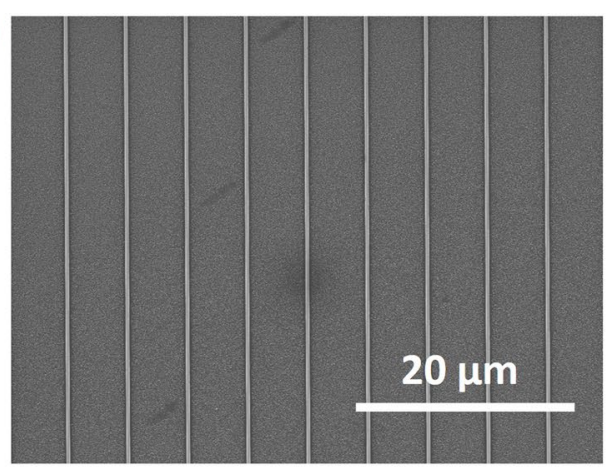

Figure 4. Variable height structures. (a) Height data obtained from diffractometry from samples $\mathrm{H} 1, \mathrm{H} 2$ and $\mathrm{H} 3$, stitched together at displacement 2 and $4 \mathrm{~mm}$. The black points represent the average value of height obtained by stylus profilometry, $430 \mathrm{~nm}, 305 \mathrm{~nm}$ and $155 \mathrm{~nm}$ respectively. (b) Top-down SEM image of sample H1.

(a)

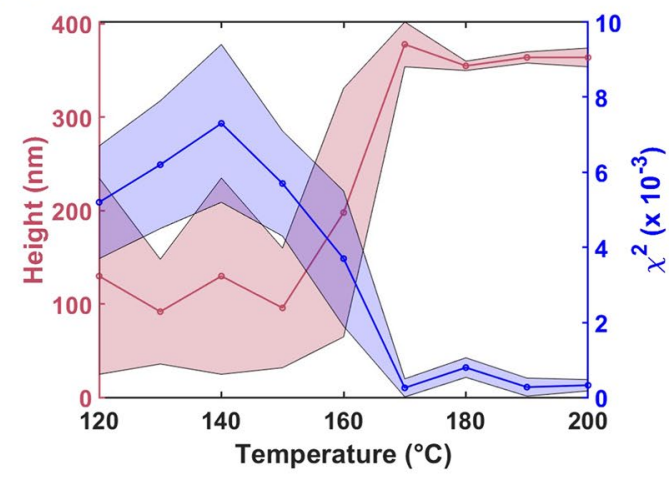

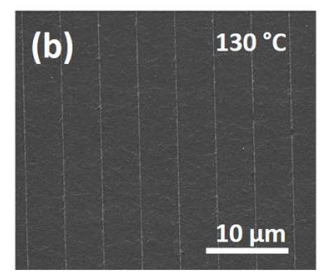

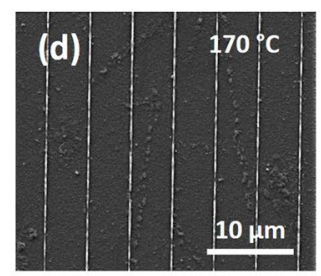

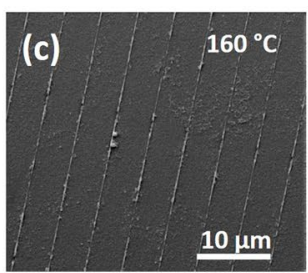

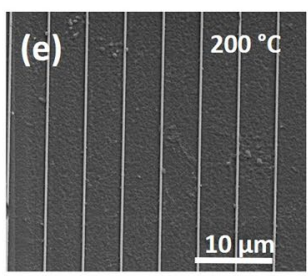

Figure 5. Thermally nanoimprinted structures. (a) Average height diffractometry data from the thermal nanoimprinted samples as a function of imprinting temperature (black) and the average minimum $\chi^{2}$ (red). (b-e) SEM image selection from samples imprinted at $130,160,170$ and $200^{\circ} \mathrm{C}$, respectively.

constant at 3 minutes. This was done in order to inspect the produced structures above and below the glass transition temperature of the PMMA used $\left(113^{\circ} \mathrm{C}\right)$.

Figure 5(a) shows the average height obtained via diffractometry when the thermal NIL samples were scanned underneath the inspection beam. The error bars plotted represent the standard deviation in measured heights for the entire scan. The minimum $\chi^{2}$ value from diffraction pattern look-up process are also plotted. Above 160 ${ }^{\circ} \mathrm{C}$ there is a clear jump in the height data from a broad uncertain prediction to a narrow average height range of 355-365 nm measured across the samples. These values are consistent with the height measured by diffractometry of the master structure $\mathrm{H} 2$ as seen in Fig. 4(a). Accompanying the jump in the height there is a notable drop in the $\chi^{2}$. As the structures begin to form under sufficient temperature by the thermal NIL process, they become uniform over a larger area and exhibit fewer defects, allowing the $\chi^{2}$ minimisation process to find an appropriate diffraction pattern in the library to match to the experimental data.

A selection of SEM images was taken across the sample set (shown in Fig. 5(b-e)) of samples imprinted at $130,160,170$ and $200{ }^{\circ} \mathrm{C}$ respectively. These SEM images reveal that for an imprinting temperature of $160^{\circ} \mathrm{C}$ and below, the PMMA structures are not well formed and contain many defects. Above this temperature the structures become better defined which coincides with the diffractometry results, and additionally, supports the fabrication rule of thermal NIL that the imprinting temperature should be around $50^{\circ} \mathrm{C}$ above the glass transition temperature $\left(\mathrm{T}_{\mathrm{g}}=113^{\circ} \mathrm{C}\right)$ for high fidelity.

\section{Discussion}

In summary, we present a yet unexplored form of optical scatterometry for critical dimension metrology with nanoscale feature sizes; diffractometry. The technique works on the principle that the optical diffraction pattern can be used to determine the unit cell critical dimensions via inverse problem-solving whereby an imaged diffraction pattern is compared to a theoretically computed library and a $\chi^{2}$ minimisation process is used to predict the 
structural parameters in real-time. The method was tested on different silicon and PMMA structures fabricated by e-beam lithography and thermal nanoimprint lithography respectively. Additionally, thermal NIL samples fabricated under various imprinting temperatures were inspected, demonstrating the viability of diffractometry as quality control for nanoimprint lithography.

The tool was used to detect the variation of structural parameters of linear grating; height, width and side-wall angle. Diffractometry showed an extremely high sensitivity to structural and surface defects making it easy to identify high fidelity areas and those with a higher degree of defectivity. Averaged across all sample sets, the top-width and height measurements via diffractometry were accurate to $9.1 \%$ and $9.8 \%$ when compared to SEM images and stylus profilometry as references respectively. In most cases this equated to several tens of nanometres difference and is on the same order as the uncertainty of the cross-referencing method. Side-wall angles measurements however exhibited a much greater level of inaccuracy averaging to a $25 \%$ difference from those calculated via the combination of SEM and profilometry. This is believed to be due to the FDFD model not taking into account corner rounding and over-etching.

This technique differs from other forms of scatterometry in a variety of different ways. Principally this technique uses the diffraction efficiency as a function of diffraction order as opposed to wavelength or angle-of-incidence. As such a different computational technique had to be used to accurately predict all higher order orders of diffraction, namely, finite-difference frequency-domain (FDFD). FDFD has the advantage of being able to simulate realistic experimental conditions whereby a finite structure is computed with a Gaussian incident field. Future improvements can be made by moving to finite-element methods (FEM) which allow for accurate simulation of curved edges and slopped edges potentially improving the accuracy of the computational libraries.

Further to the presented work we have made initial attempts to integrate real-time diffractometry in-line to a UV-assisted roll-to-roll nanoimprint, laboratory scale tool. This has proved promising although technical issues of stability during the roll-to-roll NIL process have yet to be solved. We envisage diffractometry to become an important quality control inspection technique for high-volume microstructure production giving critical dimension accuracy down to $\sim 10 \mathrm{~nm}$.

\section{Materials and Methods}

Optical set-up. The optical set-up consisted of a fibre-coupled HeNe source collimated by an elliptical mirror, subsequently polarised to match the simulations, and then telescoped down to beam-size of $200 \mu \mathrm{m}$ onto the sample stage, as shown in Fig. 1(a). The sample plane was positioned one focal length away from the aspherical objective $(16 \mathrm{~mm})$. The sample stage was motorised to control the scanning speed and to mimic a real-time inspection in a production line. All samples were measured at $1 \mathrm{~mm} / \mathrm{s} \mathrm{scanning} \mathrm{speed.} \mathrm{The} \mathrm{CCD} \mathrm{camera} \mathrm{is} \mathrm{positioned} \mathrm{in}$ the Fourier plane of the sample stage in order to obtain a sharp diffraction pattern. The speed of measurement was limited to $100 \mathrm{~ms}$, due to the internal $10 \mathrm{fps}$ frame rate of the camera. The photodiode was positioned in a collimated section of the 0 th reflected beam. Higher orders diffraction travelling off the optical axis were blocked off by a pin hole such that only the $0^{\text {th }}$ order was detected by the photodiode. The power measured from the photodiode was then used to normalise the data collected from the CCD such that the $0^{\text {th }}$ order diffraction was equal to unity (interfaced via LabVIEW). The analysed CCD images there for provides a one-dimensional array containing the diffraction intensity with respective to the $0^{\text {th }}$ order of each detected higher order of diffraction.

Real-time metrology. The normalised observed diffraction efficiencies $\left(O_{i}^{\text {norm }}\right)$ from the CCD were fed into a simultaneously running MATLAB code which was used to compare the observed values to those in the EM library $\left(E x_{i}\right)$. For each cell in the library a chi-squared value was calculated:

$$
\chi^{2}=\sum_{i} \frac{\left(O_{i}^{\text {norm }}-E x_{i}\right)^{2}}{E x_{i}}
$$

where $i$ denotes the order of diffraction.

The minimum value of in the generated chi-squared array was then located by converting the $\mathrm{n}$-dimensional array into a $1 \mathrm{D}$ array, extracting the minimum value, and then reconverting it's index back into the original parametric dimensions to identify the corresponding top width, height and SWA parameters from the EM-library. These are then given as the measured result of said parameter (solid coloured lines in Figs. 2(b,c), 3(b) and 4(a)). Additionally, the parameters for chi-squared cells within the relative experimental uncertainty of the minimum were generated to give an idea of experimental uncertainty in the measurement (filled circles in Figs. 2(b,c), 3(b) and $4(\mathrm{a}))$.

Due to the asymmetrical nature of the tapered structures, the diffraction patterns are degenerate for the first and second half of the grating lines. In order to measure the entire length of the structure two diffraction libraries had to be used, one for the first half, and another for the second; the two sets of results were later stitched together for presentation purposes at $35 \mathrm{~mm}$ displacement in Fig. 3(b).

Stylus profilometry. To obtain a close approximation of the height for these thin films $(\sim 100 \mathrm{~nm})$, a stylus profilometer (Alphastep D500, KLA Tencor inc.) was used for scanning at a near parallel angle to the grating lines, $\sim 5^{\circ}$ off axis. In this manner we could maximise the time spent by the scanning tip on the thin structures to get an accurate height reading. Attempts were made to use AFM for this cross reference, however, the height of the structures made accurate readings problematic. 
Si grating fabrication. The silicon structures were fabricated by electron beam lithography using a Vistec EBPG5000 system. A $550 \mathrm{~nm}$ thick PMMA 996k film was spin coated on a HMDS coated 4-inch Si wafer followed by a 6 minute pre-bake at $180^{\circ} \mathrm{C}$. A beam step size of $25 \mathrm{~nm}$ including proximity effect correction was used to achieve a quality of side wall roughness. Reactive ion etching was carried out to transfer the pattern into the $\mathrm{Si}$ substrate to the desired height.

Thermal nanoimprint lithography. The thermal nanoimprint lithography (NIL) process was performed using a desktop equipment (CNI Tool from NIL Technology ApS), which allows imprinting of any stamp onto a substrate size of up to $10 \mathrm{~cm}$ in diameter. The substrates were $2 \times 2 \mathrm{~cm}^{2}$ PMMA films with a thickness of $125 \mu \mathrm{m}$. Before the thermal NIL process, the silicon master stamp was treated with an anti-adhesion material, Optool ${ }^{\mathrm{TM}}$ DSX from Daikin Chemical Europe (Düsseldorf, Germany), in order to prevent possible adhesion. All samples were patterned using an imprinting time of $3 \mathrm{~min}$ and a pressure of $5 \mathrm{bar}$, while the imprinting temperature was varied from $120^{\circ} \mathrm{C}$ to $200^{\circ} \mathrm{C}$ in $10^{\circ} \mathrm{C}$ steps; the separation temperature between stamp and substrate was fixed at $40^{\circ} \mathrm{C}$

Received: 18 October 2019; Accepted: 27 January 2020;

Published online: 25 March 2020

\section{References}

1. Sreenivasan, S. V. Nanoimprint lithography steppers for volume fabrication of leading-edge semiconductor integrated circuits. Microsystems Nanoeng. 3, 17075 (2017).

2. Javidi, B. et al. Roadmap on optical security. J. Opt. (United Kingdom) 18, 1-39 (2016).

3. Cadarso, V. J., Chidambaram, N., Jacot-Descombes, L. \& Schift, H. High-aspect-ratio nanoimprint process chains. Microsystems Nanoeng. 3, 17017 (2017)

4. Yang, H. \& Gijs, M. A. M. Micro-optics for microfluidic analytical applications. Chem. Soc. Rev. 47, 1391-1458 (2018).

5. Kim, S. et al. Nanostructured multifunctional surface with antireflective and antimicrobial characteristics. ACS Appl. Mater. Interfaces 7, 326-331 (2015).

6. Department of Energy. Roll to Roll Processing (2015).

7. Leitgeb, M. et al. Multilength Scale Patterning of Functional Layers by Roll-to-Roll Ultraviolet-Light-Assisted Nanoimprint Lithography. ACS Nano 10, 4926-4941 (2016).

8. Morse, J. D. Nanofabrication Technologies for Roll-to-Roll Processing. NIST-NNN Work. 1-32 (2011).

9. Kooy, N., Mohamed, K., Pin, L. T. \& Guan, O. S. A review of roll-to-roll nanoimprint lithography. Nanoscale Res. Lett. 9, 320 (2014).

10. Bugnicourt, E. et al. Recent Prospects in the Inline Monitoring of Nanocomposites and Nanocoatings by Optical Technologies. Nanomaterials 6, 150 (2016).

11. Bogue, R. Nanometrology: a critical discipline for the twenty-first century. Sens. Rev. 27, 189-196 (2007).

12. Leach, R. K. et al. The European nanometrology landscape. Nanotechnology 22, 062001 (2011).

13. Subbaraman, H. et al. Metrology and Instrumentation Challenges with High-rate, Roll-to-Roll Manufacturing of Flexible Electronic Systems. Instrumentation, Metrol. Stand. Nanomanufacturing, Opt. Semicond. VI 8466, 846603 (2012).

14. Coates, P. D. et al. In-process vibrational spectroscopy and ultrasound measurements in polymer melt extrusion. Polymer (Guildf). 44, 5937-5949 (2003)

15. Fischer, D. et al. Process monitoring of polymers by in-line ATR-IR, NIR and Raman spectroscopy and ultrasonic measurements. Comptes Rendus Chim. 9, 1419-1424 (2006).

16. Kumar, N. et al. Coherent Fourier scatterometry: tool for improved sensitivity in semiconductor metrology. SPIE Adv. Lithogr. 83240Q, https://doi.org/10.1117/12.916357 (2012).

17. Madsen, M. H. \& Hansen, P. E. Scatterometry-fast and robust measurements of nano-textured surfaces. Surface Topography: Metrology and Properties 4 (2016).

18. Gawhary, O., Kumar, N., Pereira, S. F., Coene, W. M. J. \& Urbach, H. P. Performance analysis of coherent optical scatterometry. Appl. Phys. B 105, 775-781 (2011).

19. Losurdo, M. Applications of ellipsometry in nanoscale science: Needs, status, achievements and future challenges. Thin Solid Films 519, 2575-2583 (2011).

20. Madsen, J. S. et al. Scatterometry for optimization of injection molded nanostructures at the fabrication line. Int. J. Adv. Manuf. Technol. 99, 2669-2676 (2018).

21. Madsen, J. S. et al. In-line characterization of nanostructured mass-produced polymer components using scatterometry. J. Micromechanics Microengineering 27 (2017).

22. Madsen, M. H., Hansen, P.-E., Zalkovskij, M., Karamehmedović, M. \& Garnæs, J. Fast characterization of moving samples with nano-textured surfaces. Optica 2, 301 (2015).

23. Bodermann, B., Ehret, G., Endres, J. \& Wurm, M. Optical dimensional metrology at Physikalisch-Technische Bundesanstalt (PTB) on deep sub-wavelength nanostructured surfaces. Surf. Topogr. Metrol. Prop. 4 (2016).

24. Raymond, C. Overview of scatterometry applications in high volume silicon manufacturing. In AIP Conference Proceedings 788, 394-402 (2005).

25. Wurm, M., Pilarski, F. \& Bodermann, B. A new flexible scatterometer for critical dimension metrology. Rev. Sci. Instrum. 81 (2010).

26. Al-Assaad, R. M., Regonda, S., Tao, L., Pang, S. W. \& Hu, W. (Walter). Characterizing nanoimprint profile shape and polymer flow behavior using visible light angular scatterometry. J. Vac. Sci. Technol. B Microelectron. Nanom. Struct. 25, 2396 (2007).

27. Roy, S., Assafrão, A. C., Pereira, S. F. \& Urbach, H. P. Coherent Fourier scatterometry for detection of nanometer-sized particles on a planar substrate surface. Opt. Express 22, 13250-62 (2014).

28. Kreuzer, M. et al. In-line metrology for roll-to-roll UV assisted nanoimprint lithography using diffractometry. APL Mater. 6 (2018).

29. Kreuzer, M., Gomis Bresco, J., Sledzinska, M. \& Sotomayor-Torres, C. M. In-line metrology setup for periodic nanostructures based on sub-wavelength diffraction. Proc. SPIE 9628, Opt. Syst. Des. 2015 Opt. Fabr. Testing, Metrol. V 96281Q, https://doi. org/10.1117/12.2191346 (2015).

30. Schneider, J. B. Near-to-Far-Field Transformation. In Understanding the Finite-Difference Time-Domain Method 351-376 (2010).

\section{Acknowledgements}

The authors acknowledge the financial support from the EU project, FLEXPOL (No. H2020-NMBPPILOT-2016-721062) and the project: Surface and interface reshaped phonon propagation and phonon coupling to photons (SIP). PGC2018-101743-B-I00 (MINECO). The ICN2 is funded by the CERCA programme/ Generalitat de Catalunya and is supported by the Severo Ochoa programme of the Spanish Ministry of Economy, Industry and Competitiveness (MINECO, grant no. SEV-2017-0706). 


\section{Author contributions}

C.M.S.T. and N.K. conceived of the original diffractometry concept and supervised the work. N.K. additionally fabricated all silicon master structures via electron beam lithography. Thermal nanoimprinting was carried out by A.F. All other aspects of the work such as the design, experimentation, computational framework and the preparation of the manuscript/figures were performed by G.L.W. The manuscript was reviewed by all authors.

\section{Competing interests}

The authors declare no competing interests.

\section{Additional information}

Supplementary information is available for this paper at https://doi.org/10.1038/s41598-020-61975-3.

Correspondence and requests for materials should be addressed to G.L.W.

Reprints and permissions information is available at www.nature.com/reprints.

Publisher's note Springer Nature remains neutral with regard to jurisdictional claims in published maps and institutional affiliations.

(c) (i) Open Access This article is licensed under a Creative Commons Attribution 4.0 International

License, which permits use, sharing, adaptation, distribution and reproduction in any medium or format, as long as you give appropriate credit to the original author(s) and the source, provide a link to the Creative Commons license, and indicate if changes were made. The images or other third party material in this article are included in the article's Creative Commons license, unless indicated otherwise in a credit line to the material. If material is not included in the article's Creative Commons license and your intended use is not permitted by statutory regulation or exceeds the permitted use, you will need to obtain permission directly from the copyright holder. To view a copy of this license, visit http://creativecommons.org/licenses/by/4.0/.

(c) The Author(s) 2020 